\title{
Delayed peripartum cardiomyopathy after emergency cesarean section
}

\author{
Jung-Won Kim, Woo-Jong Choi, Jeong-Hun Suh, Sung-Kang Cho, and Sung Min Han \\ Department of Anesthesiology and Pain Medicine, Asan Medical Center, University of Ulsan College of Medicine, Seoul, Korea
}

Peripartum cardiomyopathy (PPCM) is a primary myocardial disease without any demonstrable cause and with onset in the last month of pregnancy or within six months of delivery [1]. Mortality is as high as 20 to $50 \%$ [2]. PPCM may suddenly occur when medical monitoring has been neglected and should be managed with great care by anesthesiologists and obstetricians.

A 40-year-old (weight $81.5 \mathrm{~kg}$, height $157 \mathrm{~cm}$ ) multigravida (1-0-1-1) was admitted at 34 weeks 1 day of gestation with a chief complaint of preterm labor. Her medical history included obesity and gestational diabetes mellitus in a previous pregnancy. At 32 weeks gestation, she developed gestational hypertension without other significant preeclampsia signs and symptoms. At the time of admission the patient had a blood pressure (BP) of 180/100 mmHg, heart rate (HR) of 106 beats/ min and respiratory rate (RR) of 20 breaths/min. She was diagnosed with pregnancy-induced hypertension, preterm labor occurred so emergency cesarean section was decided. Physical examination yielded no specific findings. In blood tests hemoglobin was $14.5 \mathrm{~g} / \mathrm{dl}$, hematocrit $40.9 \%$, and coagulation and electrolyte were within normal range, as were chest x-ray and electrocardiogram (ECG). Hence combined spinal-epidural anesthesia was planned.

The patient arrived in the operating room with premedication and antacids. ECG, non-invasive blood pressure and pulse oxymetry were monitored. Vital signs were BP 178/105 mmHg, HR 112 beats/min, RR 20 breaths/min and oxygen saturation $96 \%$. Oxygen was administered via nasal prong at 2 $\mathrm{L} /$ min. Combined spinal epidural technique (Portex ${ }^{\circledR}$, Smith Medical International Ltd, Kent, UK) with $7 \mathrm{mg}$ of $0.5 \%$ heavy bupivacaine and $15 \mu \mathrm{g}$ of fentanyl was done in left lateral decubitus position and midline approach at L3-4 interspace. Level of block was checked after 5 and 10 minutes and was found to be up to thoracic $6^{\text {th }}$ and $4^{\text {th }}$ segment respectively. Surgery proceeded without event and a healthy baby was delivered. Intravenous Carbetocin (Duratocin ${ }^{\circledR}$, Ferring International Center, Switzerland) $100 \mu \mathrm{g}$ and midazolam $2 \mathrm{mg}$ were given after delivery. Total estimated blood loss was 700 $\mathrm{ml}$. The patient received 2,000 $\mathrm{ml}$ of lactated Ringer's solution, and urine output was $200 \mathrm{ml}$. The operation lasted 65 minutes without adverse hemodynamic events.

Patient was transferred to the postanesthetic care unit and then, over an hour later, to general ward, with stable vital signs. On POD 2, the patient's general condition was good but as her BP was 160-180/90-110 mmHg, anti-hypertensive medication was given. She was supposed to be discharged on POD 4, but suddenly had chest pain and dyspnea, and oxygen saturation by pulse oxymetry became unstable as $81 \%$. Accordingly, oxygen was administered via a reservoir mask, but oxygen saturation continued to be unstable. Immediately, endotracheal intubation and cardiopulmonary cerebral resuscitation were performed and the patient was transferred to the intensive care unit. On chest $\mathrm{x}$-ray the patient had both pulmonary edema and cardiomegaly. Arterial blood gas analysis $\left(\mathrm{FiO}_{2} \mathrm{0.6}\right)$ gave $\mathrm{pH}$ of $7.24, \mathrm{CO}_{2}$ of $50 \mathrm{mmHg}, \mathrm{O}_{2}$ of $66 \mathrm{mmHg}$, bicarbonate of $22 \mathrm{mEq} /$ $\mathrm{L}$, base excess of $-6.0 \mathrm{mEq} / \mathrm{L}$ and oxygen saturation of $95 \%$. Laboratory results at that time were B-type natriuretic peptide (BNP) 2,114 pg/ml and D-dimer $3.18 \mathrm{pg} / \mathrm{ml}$; other laboratory tests were within the normal range. A computed tomography

Corresponding author: Woo-Jong Choi, M.D., Ph.D., Department of Anesthesiology and Pain Medicine, Asan Medical Center, University of Ulsan College of Medicine, Pungnap-dong, Songpa-gu, Seoul 138-736, Korea. Tel: 82-2-3010-3868, Fax: 82-2-3010-6790, E-mail: azac2000@naver.com (c) This is an open-access article distributed under the terms of the Creative Commons Attribution Non-Commercial License (http:// creativecommons.org/licenses/by-nc/3.0/), which permits unrestricted non-commercial use, distribution, and reproduction in any medium, provided the original work is properly cited. 


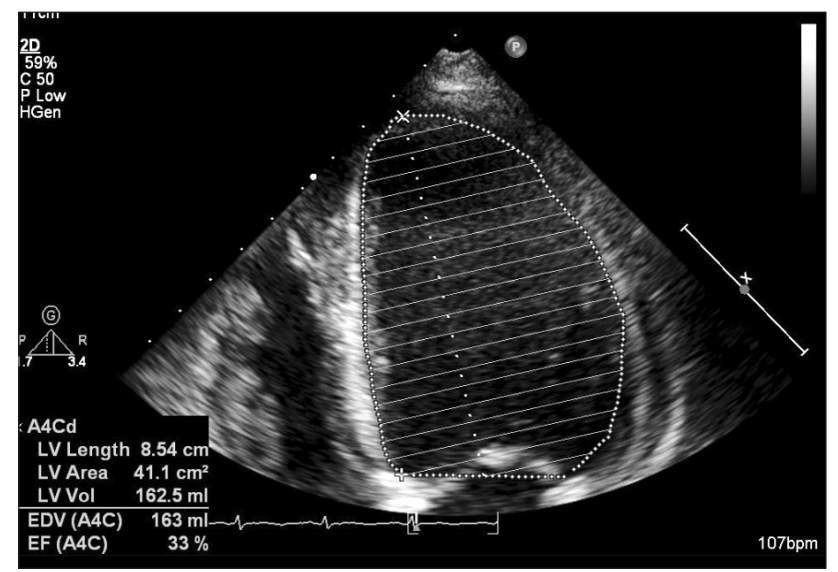

Fig. 1. A transthoracic echocardiography revealed a left ventricular ejection fraction of $33 \%$, and severe left ventricular dysfunction with mild mitral regurgitation.

chest scan to test for possible pulmonary emboli revealed pulmonary congestion and cardiomegaly but no pulmonary emboli. A transthoracic echocardiography (TTE) revealed a left ventricular ejection fraction of $33 \%$, and severe left ventricular dysfunction with mild mitral regurgitation (Fig. 1). Therefore, she was given dobutamine, heparin and furosemide under a diagnosis of PPCM with acute pulmonary edema. On POD 6, her vital signs were BP 150/95 mmHg, HR 105 beats/min, RR 20 breaths/min and oxygen saturation of $96-99 \%$. As her symptoms had improved her endotracheal tube was removed. On POD 7, vital signs remained stable. Arterial blood gas analysis $\left(\mathrm{FiO}_{2} 0.4\right)$ gave a $\mathrm{pH}$ of $7.48, \mathrm{CO}_{2}$ of $32.5 \mathrm{mmHg}, \mathrm{O}_{2}$ of $139.7 \mathrm{mmHg}$, bicarbonate of $22.7 \mathrm{mEq} / \mathrm{L}$, base excess of -1.6 $\mathrm{mEq} / \mathrm{L}$ and oxygen saturation of $97 \%$. TTE done on the same day showed a left ventricular ejection fraction of $36 \%$. Since left ventricular ejection fraction improved slightly compared with POD 4, the patient was transferred to a general ward. On POD 13 the patient was discharged without symptoms, and a followup examination was scheduled for 6 month later.

The clinical features of PPCM include symptoms of congestive heart failure and chest pain [1]. Signs can include hypertension, tachycardia, tachypnea, pulmonary rales, S 3 heart sound and pedal edema. Because these symptoms and signs overlap with many other conditions ranging from normal pregnancy to pulmonary thromboembolism, diagnosis is often delayed and the disorder is under-recognized. Echocardiography is the most important diagnostic method and also provides information on the severity of peripartum cardiomyopathy and its prognosis [3]. Our patient did not complain of any particular symptoms until POD 3; symptoms such as dyspnea and chest pain occurred on POD 4 and then suddenly worsened. PPCM usually presents in the postpartum period when physiologic changes of pregnancy should be normalizing [4]. In most cases, PPCM was occurred during cesarean section or immediate postoperative period. However, our patient who developed PPCM on POD 4 could be regarded as a delayed type. Also, symptoms of PPCM overlap with many other condition and these were suddenly occurred and aggravated at any time. Therefore, PPCM should be evaluated early and exclude pulmonary thromboembolism when symptoms such as congestive heart failure occur in the postpartum period.

Risk factors include advanced maternal age, multiparity, multiple gestation, obesity, gestational hypertension, preeclampsia and black race [1]. The prognosis of PPCM is related to its presentation as well as to recovery of ventricular dysfunction [1]. The ejection fraction normalizes in about $50 \%$ of patients. However, a second pregnancy is usually not recommended, because PPCM recurs in more than $30 \%$ [5].

In conclusion, delayed PPCM can be occurred in the patients with advanced maternal age, obesity and gestational hypertension. Therefore, these patients should be provided with adequate preoperative optimization using a multidisciplinary approach, proper use of anesthetic technique, careful intraoperative monitoring as well as vigilant postoperative care.

\section{References}

1. Bhakta P, Mishra P, Bakshi A, Langer V. Case report and mini literature review: anesthetic management for severe peripartum cardiomyopathy complicated with preeclampsia using sufentanil in combined spinal epidural anesthesia. Yonsei Med J 2011; 52: 1-12.

2. Abboud J, Murad Y, Chen-Scarabelli C, Saravolatz L, Scarabelli TM. Peripartum cardiomyopathy: a comprehensive review. Int J Cardiol 2007; 118: 295-303.

3. Heider AL, Kuller JA, Strauss RA,Wells SR. Peripartum cardiomyopathy: a review of the literature. Obstet Gynecol Surv 1999; 54: 526-31.

4. Demakis JG, Rahimtoola SH. Peripartum cardiomyopathy. Circulation 1971; 44: 964-8.

5. Wang M. Peripartum cardiomyopathy: case reports. Perm J 2009; 13: $42-5$. 